\title{
Administration of two probiotic strains during early childhood does not affect the endogenous gut microbiota composition despite probiotic proliferation
}

Martin Frederik Laursen', Rikke Pilmann Laursen², Anni Larnkjær², Kim F. Michaelsen², Martin lain Bahl'1 and Tine Rask Licht ${ }^{1 *}$ (D)

\begin{abstract}
Background: Probiotics are increasingly applied to prevent and treat a range of infectious, immune related and gastrointestinal diseases. Despite this, the mechanisms behind the putative effects of probiotics are poorly understood. One of the suggested modes of probiotic action is modulation of the endogenous gut microbiota, however probiotic intervention studies in adults have failed to show significant effects on gut microbiota composition. The gut microbiota of young children is known to be unstable and more responsive to external factors than that of adults. Therefore, potential effects of probiotic intervention on gut microbiota may be easier detectable in early life. We thus investigated the effects of a 6 month placebo-controlled probiotic intervention with Bifidobacterium animalis subsp. lactis (BB-12 ${ }^{\circledast}$ ) and Lactobacillus rhamnosus (LGG $\left.{ }^{\odot}\right)$ on gut microbiota composition and diversity in more than 200 Danish infants ( $N=290$ enrolled; $N=201$ all samples analyzed), as assessed by 165 rRNA amplicon sequencing. Further, we evaluated probiotic presence and proliferation by use of specific quantitative polymerase chain reaction (qPCR).
\end{abstract}

Results: Probiotic administration did not significantly alter gut microbiota community structure or diversity as compared to placebo. The probiotic strains were detected in $91.3 \%$ of the fecal samples from children receiving probiotics and in $1 \%$ of the placebo treated children. Baseline gut microbiota was not found to predict the ability of probiotics to establish in the gut after the 6 month intervention. Within the probiotics group, proliferation of the strains LGG ${ }^{\circledast}$ and BB-12 $2^{\circledast}$ in the gut was detected in $44.7 \%$ and $83.5 \%$ of the participants, respectively. A sub-analysis of the gut microbiota including only individuals with detected growth of the probiotics $L G G^{\oplus}$ or BB-12 ${ }^{\oplus}$ and comparing these to placebo revealed no differences in community structure or diversity.

Conclusion: Six months of probiotic administration during early life did not change gut microbiota community structure or diversity, despite active proliferation of the administered probiotic strains. Therefore, alteration of the healthy infant gut microbiota is not likely to be a prominent mechanism by which these specific probiotics works to exert beneficial effects on host health.

Trial registration: NCT02180581. Registered 30 June 2014.

Keywords: Probiotic intervention, LGG ${ }^{\oplus}, B B-12^{\circledR}$, Early life, Gut microbiota

\footnotetext{
* Correspondence: trli@food.dtu.dk

${ }^{1}$ National Food Institute, Technical University of Denmark, Kemitorvet,

DK-2800 Lyngby, Denmark

Full list of author information is available at the end of the article
} 


\section{Background}

Probiotics are defined as "live microorganisms which, when administered in adequate amounts, confer a health benefit on the host" [1]. Several mechanisms behind probiotic effects have been suggested, including modulation of the endogenous gut microbiota [2-4]. However, a recent meta-analysis of seven probiotic intervention studies showed that probiotic administration did not affect the adult gut microbiota as determined by analysis of fecal samples [5]. Still, in infants and children, the microbiota is known to be much more variable [6] and easier to manipulate [7] than seen for adults. Thus, if alteration of the gut microbiota composition is a mechanism by which probiotics exert their beneficial effect, this would be expected to be evident in early life interventions. In the present study, we have analyzed the fecal microbiota of healthy infants at the age of starting daycare (age 8-13 months), sampled before and after a 6 months intervention with a single daily dose of Lactobacillus rhamnosus (LGG ${ }^{\circledast}$ ) and Bifidobacterium animalis subsp. lactis $\left(\mathrm{BB}-12^{\circ}\right)$ or placebo. Additionally, in contrast to previous studies, we specifically assessed the presence and proliferation of the probiotic strains in the gut, and evaluated the effect on the endogenous microbial composition and diversity based on probiotic propagation.

\section{Methods \\ Study participants}

Infants, aged 8-14 months and starting daycare within 12 weeks after intervention start, were included in the ProbiComp study [8], a double blinded randomized placebo-controlled intervention. BB- $12^{\circ}$ and $\mathrm{LGG}^{\odot}$ are registered trademarks of $\mathrm{Chr}$. Hansen A/S who kindly provided the study products. During the 6 months intervention period participants were randomized to receive either a combination of the two probiotics BB- $12^{\circ}$ and $\mathrm{LGG}^{\odot}$ at a dose of $10^{9}$ colony-forming units (CFU) each, or a placebo (maltodextrin), during the winter season. Participants were instructed not to consume food products and supplements containing probiotics 2 weeks prior to and during the intervention period. The study spanned two consecutive winter seasons and included a total of 290 infants (145 for each season). Study population characteristics (Table 1) were obtained through parental interviews.

Table 1 Characteristics of study participants with gut microbiota data completing the study $(n=201)$

\begin{tabular}{|c|c|c|c|}
\hline Parameter & $\begin{array}{l}\text { Probiotics group } \\
(n=103)\end{array}$ & $\begin{array}{l}\text { Placebo group } \\
(n=98)\end{array}$ & $P$-value \\
\hline \multicolumn{4}{|l|}{ Gender } \\
\hline Girls (\%) & 45.6 & 46.9 & \multirow[t]{2}{*}{$0.888^{\mathrm{a}}$} \\
\hline Boys (\%) & 54.4 & 53.1 & \\
\hline \multicolumn{4}{|l|}{ Mode of delivery } \\
\hline C-section (\%) & 23.3 & 15.3 & \multirow[t]{2}{*}{$0.159^{a}$} \\
\hline Vaginal (\%) & 76.7 & 84.7 & \\
\hline \multicolumn{4}{|l|}{ Older siblings } \\
\hline Siblings (\%) & 48.5 & 43.9 & \multirow[t]{2}{*}{$0.572^{a}$} \\
\hline No siblings (\%) & 51.5 & 56.1 & \\
\hline \multicolumn{4}{|l|}{ Introduction of selected foods } \\
\hline Cow's milk (median months, IQR) & $8.0(7.0-12.0)$ & $9.0(6.0-11.0)$ & $0.947^{b}$ \\
\hline Meat (median months, IQR) & $6.0(6.0-7.0)$ & $6.0(6.0-7.0)$ & $0.242^{b}$ \\
\hline Fish (median months, IQR) & $6.0(6.0-7.0)$ & $6.0(6.0-7.0)$ & $0.544^{\mathrm{b}}$ \\
\hline \multicolumn{4}{|l|}{ Breastfeeding prevalence } \\
\hline 1st visit (\%) & 54.4 & 46.9 & $0.325^{\mathrm{a}}$ \\
\hline 2nd visit (\%) & 13.6 & 9.2 & $0.380^{\mathrm{a}}$ \\
\hline \multicolumn{4}{|l|}{ Age } \\
\hline 1st visit (median months, IQR) & $10.0(9.5-10.4)$ & $9.8(9.5-10.4)$ & $0.462^{\mathrm{b}}$ \\
\hline Start daycare (median months, IQR) & $10.4(9.9-11.2)$ & $10.3(9.9-11.1)$ & $0.309^{b}$ \\
\hline 2nd visit (median months, IQR) & $16.1(15.6-16.5)$ & $16.0(15.6-16.4)$ & $0.510^{b}$ \\
\hline \multicolumn{4}{|l|}{ Compliance } \\
\hline 2nd visit (median \% of consumed sticks, IQR) & 97 (93-99) & $98(94-100)$ & $0.417^{\mathrm{b}}$ \\
\hline
\end{tabular}

${ }^{\text {a }}$ ishers exact test

${ }^{\mathrm{b}}$ Mann Whitney test 
Fecal samples, DNA extraction and PCR amplification of the $\mathrm{V} 3$ region of the $16 \mathrm{~S}$ rRNA gene

Fecal samples obtained before $(N=265$, age $8-13$ months $)$ and after ( $N=210$, age 14-19 months) the intervention were freshly delivered on the morning of the visit or had been stored in the participant's home, either in the freezer $\left(-18{ }^{\circ} \mathrm{C}\right)$ or in the fridge $\left(4{ }^{\circ} \mathrm{C}\right)$ for maximally $24 \mathrm{~h}$ before delivery to the University of Copenhagen, Department of Nutrition, Exercise and Sports, where they were stored at $-80{ }^{\circ} \mathrm{C}$ until DNA extraction. Extraction was done in random order, but the two samples obtained from the same child were always processed together $(N=16-20$ samples/extraction round). $250 \mathrm{mg}$ feces was applied and treated according to the protocol provided by the manufacturer (PowerLyzer ${ }^{\odot}$ PowerSoil $^{\circ}$ DNA isolation kit, MoBio 12,855-100) with minor modifications: Bead beating was performed at $30 \mathrm{cycles} / \mathrm{s}$ for $10 \mathrm{~min}$ (Retsch MM 300 mixer mill) and the initial centrifugation steps were performed at 10,000 $\mathrm{x}$ g for $3 \mathrm{~min}$, as recommended for clay matter. DNA quantity and quality was measured by NanoDrop $^{\oplus} 1000$ (ThermoFisher Scientific), yielding on average $85.0 \pm 47.1 \mathrm{ng} / \mu \mathrm{l}$ DNA with A260/A280 = $1.89 \pm 0.10$ and A260/A230 $=1.71 \pm 0.39$. The PCR amplification of the V3region of the $16 \mathrm{~S}$ rRNA gene was performed with $5 \mathrm{ng}$ community DNA as template, using $0.2 \mu$ Phusion High-Fidelity DNA polymerase (Fisher Scientific, F-553 L), $4 \mu$ l HF-buffer, $0.4 \mu \mathrm{l}$ dNTP (10 $\mathrm{mM}$ of each base), $1 \mu \mathrm{M}$ forward primer (PBU 5' -A-adapter-TCAG-barcode-CCTACGGGAGGCA GCAG-3') and $1 \mu \mathrm{M}$ reverse primer (PBR 5'-trP1-adapterATTACCGCGGCTGCTGG-3') in $20 \mu$ lotal reaction volume. Both primers included sequencing adaptors and the forward primer additionally a unique 10-12 bp barcode (Ion Xpress ${ }^{\mathrm{TM}}$ Barcode Adapters). PCR amplification included $30 \mathrm{~s}$ at $98{ }^{\circ} \mathrm{C}, 24$ cycles of $15 \mathrm{~s}$ at $98{ }^{\circ} \mathrm{C}$ and $30 \mathrm{~s}$ at $72{ }^{\circ} \mathrm{C}$, followed by $5 \mathrm{~min}$ at $72{ }^{\circ} \mathrm{C}$. PCR products were purified by use of HighPrep ${ }^{\mathrm{rm}}$ PCR Magnetic Beads (MAGBIO $\left.{ }^{\circ}, \mathrm{AC}-60005\right)$ with the 96-well magnet stand (MAGBIO ${ }^{\circ}$, MyMag 96), according to the manufacturers recommendations. DNA quantity was measured using Qubit ${ }^{\circ}$ dsDNA HS assay (Invitrogen ${ }^{\mathrm{Tm}}$, Q32851) and a total of 456 samples contained sufficient DNA for sequencing. Samples were pooled to obtain equimolar libraries containing up to 86 samples (randomized across treatment groups and age) in each library.

\section{DNA sequencing and data handling}

Sequencing of the $16 \mathrm{~S}$ rRNA amplicon libraries was performed using the Ion OneTouch ${ }^{\text {тм }}$ and Ion PGM platform with a 318-Chip v2, generating 4-5 million reads per chip with a median length of $180 \mathrm{bp}$. Sequencing data were imported into CLC Genomic Workbench (version 8.5. CLC bio, Qiagen, Aarhus, DK) and using the NGS core tools, reads were assessed for quality ( $Q C$ report), de-multiplexed according to barcode (Demultiplexing) and trimmed (Trim sequences) to remove barcodes and PCR primers (minimum alignment score 17/17, discard when both primers were not found) and to discard reads below $125 \mathrm{bp}$ and above $180 \mathrm{bp}$. Quality filtering (-fastq_filter, maxee 1.0), dereplication (-derep_fulllength), OTU clustering (-cluster_otus, minsize 4), chimera filtering (-uchime_ref, RDP_gold database), mapping of reads to OTUs (-usearch_global, id 97\%) and generation of OTU table (python, uc2otutab.py) was done according to the UPARSE pipeline [9], generating a total of 1096 non-chimeric OTUs (Operational Taxonomic Units). In QIIME [10], the OTU table was filtered (filter_otus_from_otu_table.py/filter_fasta.py) to exclude OTUs with average relative abundance below $0.005 \%$ of the total community, resulting in 330 OTUs. Taxonomy was assigned (assign_taxonomy.py), using the rdp classifier with confidence threshold 0.5 (recommended for sequences shorter than $250 \mathrm{bp}$ [11]) and the GreenGenes database v. 13.8 [12]. In addition, the taxonomy of selected OTUs was confirmed/disconfirmed by BLAST [13] search against the $16 S$ rRNA sequence database. Based on PyNAST alignment of representative OTU sequences (align_seqs.py, filter_alignment.py, default settings), including an archaea (Methanosarcina) full length $16 \mathrm{~S}$ rRNA gene sequence as outgroup for rooting, a phylogenetic tree was created with FastTree (make_phylogeny.py, default settings) and rerooted to the outgroup, which was subsequently pruned, using Dendroscope v3.5.7 [14]. Taxon abundances (average classification: Phylum: 100\%, Family: 99.9\%, Genus: 91.8\%) and alpha diversity (Shannon index, Observed OTUs, Pielou's evenness index) and beta diversity (UniFrac distances) were calculated in QIIME (summarize_taxa.py, alpha_rarefaction.py, beta_diversity_through_plots.py), with the sequencing depth rarefied to 10,000 sequences per sample for diversity analysis. OTU abundances were estimated by total sum scaling.

\section{Detection of the probiotic strains BB- $12^{\circledR}$ and LGG ${ }^{\circledR}$ Primer design, PCR and gel electrophoresis}

Primers specific for the probiotic strains were designed using the primer3 online software tool (http://bioinfo.ut.ee/ primer3-0.4.0/). Selection of primers for the $\mathrm{LGG}^{\odot}$ strain were based on a phage-related gene target sequence [15] previously reported to be strain specific [16], whereas primers for the BB- $12^{\circ}$ strain were selected within the CRISPR-Cas system [17], specifically the Type I-U CRISPRCas7 gene in $\mathrm{BB}-12^{\circ}$ (for primer sequences see Additional file 1: Table S1). Each PCR reaction contained $14.92 \mu \mathrm{l}$ DNAse-free water, $2 \mu \mathrm{l}$ 10X Accuprime PCR buffer II, $1 \mu \mathrm{l}$ forward primer $(0.5 \mu \mathrm{M}$ final conc.), $1 \mu \mathrm{l}$ reverse primer (final conc. $0.5 \mu \mathrm{M}$ ), $0.08 \mu \mathrm{l}$ AccuPrime Taq Polymerase (Invitrogen, 12,346-086) and $1 \mu$ template DNA (1 ng/ul) in a final volume of 20 ul. The PCR program (Veriti Thermal Cycler, Applied Biosystems ${ }^{\mathrm{TM}}$, 4,452,300) included 
2 min at $94{ }^{\circ} \mathrm{C}, 35$ cycles of $20 \mathrm{~s}$ at $94{ }^{\circ} \mathrm{C}, 20 \mathrm{~s}$ at $65{ }^{\circ} \mathrm{C}$ and $30 \mathrm{~s}$ at $68{ }^{\circ} \mathrm{C}$, followed by a final extension for $5 \mathrm{~min}$ at $68{ }^{\circ} \mathrm{C}$. The PCR products were separated on a $1.5 \%$ agarose gel with 1:10,000 volume SYBR safe (Bio-Rad) for $1 \mathrm{~h}$ at $100 \mathrm{~V}$ before imaging (Molecular Imager ${ }^{\oplus}$ GelDoc $^{\mathrm{TM}}$ XR System).

\section{Primer validation and probiotic quantification}

To validate primer specificity, a selection of Lactobacillus and Bifidobacterium strains were cultivated on MRS (de Man, Rogosa and Sharpe, [18]) or BSM (Sigma, 88,517) agar at $37{ }^{\circ} \mathrm{C}$ under anaerobic conditions for $72 \mathrm{~h}$ (Additional file 1: Table S2). Genomic DNA was extracted (PowerLyzer ${ }^{\circledR}$ PowerSoil $^{\circledR}$ DNA isolation kit, MoBio 12,855100) from colony material according to the protocol provided by the manufacturer and diluted to $1 \mathrm{ng} / \mu \mathrm{l}$ and used for input in the PCR. For spiking fecal samples with known quantities of the strains $\mathrm{LGG}^{\circ}$ and $\mathrm{BB}-12^{\circ}$, single colonies of these were propagated in $10 \mathrm{ml}$ MRS medium under anaerobic conditions for $48 \mathrm{~h}$ at $37{ }^{\circ} \mathrm{C}$, centrifuged at $10,000 \times \mathrm{g}$ for $5 \mathrm{~min}$ at $4{ }^{\circ} \mathrm{C}$, re-suspended in $1 \mathrm{ml}$ maximum recovery diluent (MRD, Oxoid CM0733), and 10-fold serially diluted in MRD. One hundred $\mu \mathrm{l}$ of each dilution $\left(10^{0}-10^{-7}\right)$ was spiked into $100 \mathrm{mg}$ LGG $/ \mathrm{BB}-12^{\odot}$ negative baseline fecal samples (based on specific qPCR of the probiotic strains) and also plated in duplicates (dilutions $10^{-4}-10^{-9}$ ) on MRS plates and incubated anaerobically at $37{ }^{\circ} \mathrm{C}$ for $24 \mathrm{~h}$ before CFU counting. Community DNA was extracted (PowerLyzer ${ }^{\circledR}$ PowerSoil $^{\circ}$ DNA isolation kit, MoBio 12,855-100) from each spiked samples plus none-spiked controls.

\section{Quantitative PCR}

Each quantitative PCR (qPCR) reaction contained $5 \mu \mathrm{l}$ PCR-grade water, $1 \mu \mathrm{l}$ forward primer (final conc. $0.5 \mu \mathrm{M}$ ), $1 \mu \mathrm{l}$ reverse primer (final conc. $0.5 \mu \mathrm{M}$ ), $10 \mu \mathrm{l}$ SYBR Green I Master 2X (LightCycler 480 SYBR Green I Master, Roche, 04887352001) and $2 \mu \mathrm{l}$ template DNA, in a $20 \mu \mathrm{l}$ total volume. Template DNA was either 10-fold serial dilutions of $1 \mathrm{ng} / \mu \mathrm{l}$ DNA extracted from pure cultures of $\mathrm{LGG}^{\odot}$ or $\mathrm{BB}-12^{\circ}$, community DNA isolated from fecal samples (LGG $/$ BB- $12^{\circ}$ negative) spiked with 10 -fold serial dilutions of $\mathrm{LGG}^{\oplus}$ or BB- $12^{\oplus}$ cultures, or community DNA $(5 \mathrm{ng} / \mu \mathrm{l})$ extracted from fecal samples obtained during the intervention. Triplicate samples were run on the qPCR instrument (LightCycler 480 Instrument II, Roche, 05015243001) and the program included $5 \mathrm{~min}$ pre-incubation at $95{ }^{\circ} \mathrm{C}$, followed by 45 cycles with $10 \mathrm{~s}$ at $95{ }^{\circ} \mathrm{C}, 15 \mathrm{~s}$ at $65^{\circ} \mathrm{C}$ and $15 \mathrm{~s}$ at $72{ }^{\circ} \mathrm{C}$. A melting curve analysis was subsequently performed with $5 \mathrm{~min}$ at $95^{\circ} \mathrm{C}, 1 \mathrm{~min}$ at $65^{\circ} \mathrm{C}$ and continuous temperature increase (ramp rate $0.11{ }^{\circ} \mathrm{C} / \mathrm{s}$ ) until $98{ }^{\circ} \mathrm{C}$. Data were analyzed with the LightCycler ${ }^{\oplus} 480$ Software ( $v$ 1.5) and levels of the strains $\mathrm{LGG}^{\circ}$ and $\mathrm{BB}-12^{\circ}$ in samples were quantified based on standard curves generated from extracted community DNA of fecal samples spiked with 10-fold serial diluted DNA extracted from pure cultures of $\mathrm{LGG}^{\oplus}$ or BB- $12^{\circ}$. Samples were regarded $\mathrm{LGG}^{\oplus}$ and/ or $\mathrm{BB}-12^{\circ}$ positive when at least 2 of the 3 replicates were positive, the melting curves were consistent with that of pure cultures of the respective strains, and $C_{t}$ values were within the detection range achieved for the 10-fold dilution series standard-curves.

\section{Statistics}

Statistics were performed with the GraphPad Prism software (v. 7.0, GraphPad Software Inc., La Jolla, CA), using the Spearman's rank correlation, Mann-Whitney test and Fisher's exact test or by use of specific scripts for analyzing sequencing data as implemented in QIIME v. 1.9 [10]. For comparison of beta diversity the compare_categories.py (adonis, permutations $=999$ ) script was used and for comparison of microbial composition at different taxonomical levels the group_significance.py (mann_whitney_u, Benjamini-Hochberg False Discovery Rate (FDR) correction [19], threshold 0.05) script was applied. Alpha diversity measures were compared between groups using the Mann-Whitney test of the average of 10 iterated values obtained following rarefying to 10,000 reads per sample.

\section{Results}

\section{Study population characteristics and compliance}

There were no statistically significant differences in gender, mode of delivery, age at introduction to selected foods (cow's milk, fish and meat), breastfeeding prevalence, presence of older siblings, age at daycare start or age at samplings between the probiotics and the placebo groups, thus these parameters are unlikely to have affected our results (Table 1). Median compliance to the assigned treatments was $97 \%$ and $98 \%$ in the probiotics and placebo groups, respectively (Table 1).

\section{Microbiota profiling}

Sequencing of the V3 region of the $16 \mathrm{~S}$ rRNA genes was successful for a total of 456 fecal samples collected before $(n=255)$ and after $(n=201)$ intervention, and resulted in 11.8 million trimmed and quality filtered reads. A significant shift in alpha diversity (Shannon index, $p<0.0001$, Mann Whitney) and beta diversity (Weighted UniFrac, $\mathrm{R}^{2}=0.061, p=0.001$, Adonis) was observed during the 6 months intervention period (Additional file 1: Figure S1), which confirm a strong impact of age on gut microbiota in agreement with previous reports [20, 21]. At baseline, no differences in beta diversity (Weighted UniFrac, $\mathrm{R}^{2}=0.010, p=0.226$, Adonis), or alpha diversity (Shannon index, $p=0.868$, Pielou's evenness index, $p=0.562$ and Observed OTUs, $p=0.821$ ) were observed between the treatment group and the placebo group (Additional file 1: Figure S2). Further, no OTUs were 
significantly differently abundant between the two groups after FDR correction (Additional file 1: Table S3). After the intervention, Principle Coordinate Analysis (PCoA) of both weighted and unweighted UniFrac distances did not result in visible separation of samples according to treatment group (Fig. 1a), although Adonis testing revealed a significant difference between the unweighted distances caused by the specific presence of the probiotics as described in the next section. Estimates of alpha diversity did not differ between treatment groups (Fig. 1b). Only two OTUs were found to be significantly differently abundant following FDR correction (Table 2). These were designated OTU_17 and OTU_50, and were assigned to
Bifidobacterium pseudolongum and Lactobacillus zeae respectively by the GreenGenes classifier. However, a BLAST search against the $16 \mathrm{~S}$ rRNA gene database revealed that they had $100 \%$ homology to the $\mathrm{V} 3$ regions of the two probiotic strains included in the intervention, namely B. animalis subsp. lactis and L. rhamnosus strains, respectively (Table 2 ). Testing for differential abundances at family and genus levels revealed only Lactobacillaceae/Lactobacillus as significantly higher in the probiotics group than in the placebo group (FDR adjusted $p$-value $_{\text {family }}=3.9 \times 10^{-14}$; FDR adjusted $p$-value $\left._{\text {genus }}=9.1 \times 10^{-14}\right)$, reflecting the increased abundance of the $\mathrm{LGG}^{\circ}$ strain in the probiotics group

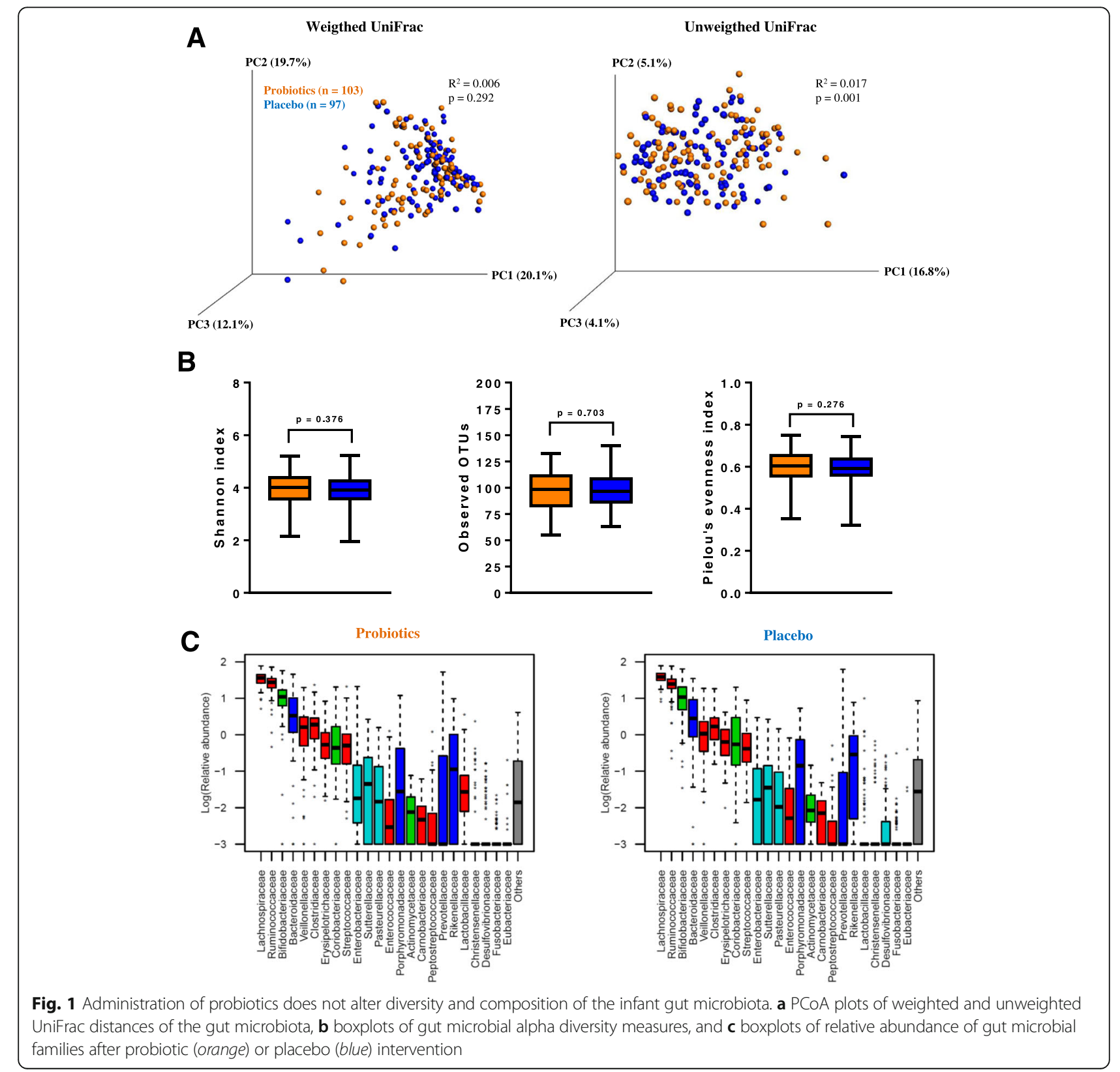


Table 2 OTUs significantly differentially abundant in probiotics $(n=103)$ vs. placebo $(n=98)$

\begin{tabular}{|c|c|c|c|c|c|}
\hline \multirow[t]{2}{*}{ OTU ID } & \multirow[t]{2}{*}{$P$-value } & \multirow{2}{*}{$\begin{array}{l}\text { FDR } \\
P \text {-value }\end{array}$} & \multicolumn{2}{|c|}{ Mean abundance (\%) } & \multirow[t]{2}{*}{ NCBI BLAST hit } \\
\hline & & & Probiotics & Placebo & \\
\hline OTU_17 & 1.45E-27 & 4.97E-25 & $2.231 \%$ & $0.018 \%$ & 100\% Bifidobacterium animalis subsp. lactis strain YIT 4121 \\
\hline OTU_50 & 4.93E-22 & $8.45 \mathrm{E}-20$ & $0.086 \%$ & $0.007 \%$ & 100\% Lactobacillus rhamnosus strain NBRC 3425 \\
\hline OTU_6 & 0.013 & 0.870 & $3.049 \%$ & $5.504 \%$ & 100\% Eubacterium rectale strain ATCC 33656 \\
\hline OTU_217 & 0.015 & 0.870 & $0.002 \%$ & $<0.001 \%$ & 99\% [Clostridium] lactatifermentans strain G17 \\
\hline OTU_3 & 0.016 & 0.870 & $5.053 \%$ & $7.642 \%$ & 100\% Bifidobacterium pseudocatenalutum strain B1279 \\
\hline OTU_290 & 0.016 & 0.870 & $0.009 \%$ & $0.004 \%$ & 97\% Butyricimonas paravirosa strain $214-4$ \\
\hline OTU_334 & 0.018 & 0.870 & $0.040 \%$ & $0.001 \%$ & 98\% Parasutterella excrementihominis strain YIT 11859 \\
\hline OTU_25 & 0.037 & 1 & $0.867 \%$ & $1.457 \%$ & 99\% Ruminococcus bromii strain ATCC 27255 \\
\hline OTU_60 & 0.041 & 1 & $0.184 \%$ & $0.117 \%$ & 100\% Barnesiella intestinihominis strain JCM 15079 \\
\hline OTU_230 & 0.048 & 1 & $0.001 \%$ & $<0.001 \%$ & 94\% Ethanoligenens harbinense strain YUAN-3 \\
\hline
\end{tabular}

(Fig. 1c). Exclusion of subjects known to have consumed infant formula containing any type of probiotics at any given point during the intervention yielded similar results.

\section{Quantification of probiotic strains in fecal samples} Design of primers specifically targeting the probiotics $\mathrm{LGG}^{\oplus}$ and $\mathrm{BB}-12^{\circ}$ (Additional file 1: Figure S3) allowed qPCR based quantification of these strains in the samples obtained after intervention. Strong correlations between qPCR-estimated abundances of the strain $\mathrm{LGG}^{\circ}$ and relative abundance of OTU_50 (rho $=0.79, p<0.0001)$ as well as between qPCR-estimated abundances of the strain BB$12^{\circ}$ and relative abundance of OTU_17 (rho $=0.89$, $p<0.0001$ ), confirmed that the two OTUs represented the two probiotic strains. When these two OTUs were omitted, an unweighted UniFrac analysis with Adonis test no longer indicated any difference between the probiotic and the placebo group $\left(R^{2}=0.004, p=0.670\right)$. By specific qPCR, both of the probiotic strains were detected in 91.3\% of the infants that received probiotics and only in $1.0 \%$ of the infants receiving placebo (Table 3 ). The strain $\mathrm{LGG}^{\oplus}$ was detected in $91.3 \%$ whereas the strain BB-12 ${ }^{\circ}$ was detected in $95.1 \%$ of the infants treated with probiotics. However, while the $\mathrm{LGG}^{\ominus}$ strain specific primers produced an amplicon in only $2.0 \%$ of the individuals receiving placebo, the $\mathrm{BB}-12^{\circ}$ strain primers, which are subspecies-specific, but not strain-specific (Additional file 1: Figure S3), amplified a target in $31.1 \%$ of the placebo treated infants (Table 3), suggesting an endogenous occurrence of

Table 3 qPCR detection of the probiotic strains in the probiotics and placebo treatment groups

\begin{tabular}{llll}
\hline & LGG & BB-12 & LGG \& BB-12 \\
& positive & positive & positive \\
\hline Probiotics $(n=103)$ & $94 / 103(91.3 \%)$ & $98 / 103(95.1 \%)$ & $94 / 103(91.3 \%)$ \\
Placebo $(n=98)$ & $2 / 98(2.0 \%)$ & $32 / 98(31.1 \%)$ & $1 / 98(1.0 \%)$ \\
Total $(n=201)$ & $96 / 201(46.8 \%)$ & $130 / 201(64.7 \%)$ & $95 / 201(47.3 \%)$ \\
\hline
\end{tabular}

B. animalis subsp. lactis as found in previous studies [22], or eventual prior ingestion of infant formula containing related strains. The qPCR-estimated abundances of the strain $\mathrm{BB}-12^{\circ}$ (range $1.3 \times 10^{4}$ to $1.5 \times 10^{10} \mathrm{CFU} / \mathrm{g}$ feces) and the strain $\mathrm{LGG}^{\oplus}\left(1.5 \times 10^{4}\right.$ to $1.4 \times 10^{9} \mathrm{CFU} / \mathrm{g}$ feces $)$ showed high inter-individual variation. Since this variation may reflect differences in colonization resistance of the endogenous microbiota, we investigated whether selected baseline gut microbiota signatures explained the variation in the quantified amounts of the probiotic strains after intervention. However, neither alpha diversity measures nor relative abundances of individual microbial taxa at baseline correlated with the qPCR detected abundances of the administered probiotics measured at the end of the intervention (data not shown).

\section{Estimation of probiotic proliferation in the infant gut}

In order to estimate whether or not the probiotic strains were proliferating in the gut, we determined the ratios between fecal excretion and oral intake of the two probiotic strains based on specific qPCR measurements for infants in the probiotic group. Intake of each of the probiotic strains, $\mathrm{LGG}^{\oplus}$ and $\mathrm{BB}-12^{\circ}$, was set as the daily dose of $10^{9} \mathrm{CFU}$. The amount of excreted probiotics was calculated as the qPCR-estimated amount of CFU/g feces multiplied with the average weight of feces reported to be excreted by healthy individuals at the given age, which is $80 \mathrm{~g} /$ day $[23,24]$. A ratio of fecal excretion to oral input (excretion/input) above 1 was considered to indicate that probiotic growth had occurred in the gut. In this context, it should be noted that a considerable fraction of probiotics are expected not to survive gastrointestinal transit, and that even a ratio just below 1 may thus imply probiotic proliferation. For the strain $\mathrm{BB}-12^{\circ}$, a ratio above 1 was found in 86 of the 103 individuals (83.5\%), resulting in a median excretion/intake ratio of 29 . While these estimates may be affected by occurrence of other $B$. animalis subsp. lactis strains (which we could not differentiate from the ingested 
BB- $12^{\circ}$ strain by qPCR), abundance of this subspecies was on average more than 100-fold lower in the placebo group (Table 2), suggesting that the other occurring subspecies of $B$. animalis subsp. lactis made only an insignificant contribution to the growth estimates. For the $\mathrm{LGG}^{\oplus}$ strain, a ratio above 1 was observed in 46 of the 103 infants (44.7\%) treated with probiotics, resulting in a median excretion/intake ratio of 0.9 . Noteworthy, all infants with a $\mathrm{LGG}^{\circ}$ ratio above 1 also had a BB- $12^{\circ}$ ratio above 1 (Fig. 2a-b). This shows that both of the probiotic strains were proliferating in the gut of at least $44.7 \%$ of the infants receiving probiotics. Comparison of the intestinal bacterial communities of the probiotics-treated infants with detected growth of either the $\mathrm{BB}-12^{\circ}$ strain $(n=86)$ or $\mathrm{LGG}^{\circ}$ strain $(n=46)$ to the placebo group $(n=97)$ did not reveal any differences in community structure assessed by weighted UniFrac distances (Fig. 2c-d), when OTU_17 and OTU_50 (representing the probiotics) were filtered out. Nor did we find significant differences in alpha diversity measures (Additional file 1: Figure S4). Finally, no differentially abundant OTUs were detected in this comparison
(Additional file 1: Table S4 \& Table S5), indicating that the composition of the endogenous microbiota was not affected despite proliferation of the probiotic strains in the infant gut.

\section{Discussion}

The bacterial strains $\mathrm{LGG}^{\circ}$ and $\mathrm{BB}-12^{\circ}$ represent some of the most commonly applied commercially available probiotics, and studies of their putative effects are therefore highly relevant. We investigated the impact of a 6 months intervention with daily doses of $10^{9} \mathrm{CFU}$ of these two probiotic strains on the gut microbiota of infants aged 8-13 months at onset. Community structure and diversity of the endogenous infant gut microbiota were not affected despite the fact that the gut microbiota is characterized by low stability and high responsiveness in early life [25]. Several comparable reports on probiotic treatments exist. For example, a previous intervention study with the probiotic strains $\mathrm{BB}-12^{\circ}, \mathrm{LGG}^{\circ}$ and $L$. acidophilus LA $-5^{\circ}$ given to pregnant women from the 36th gestation week until 3-months post-partum

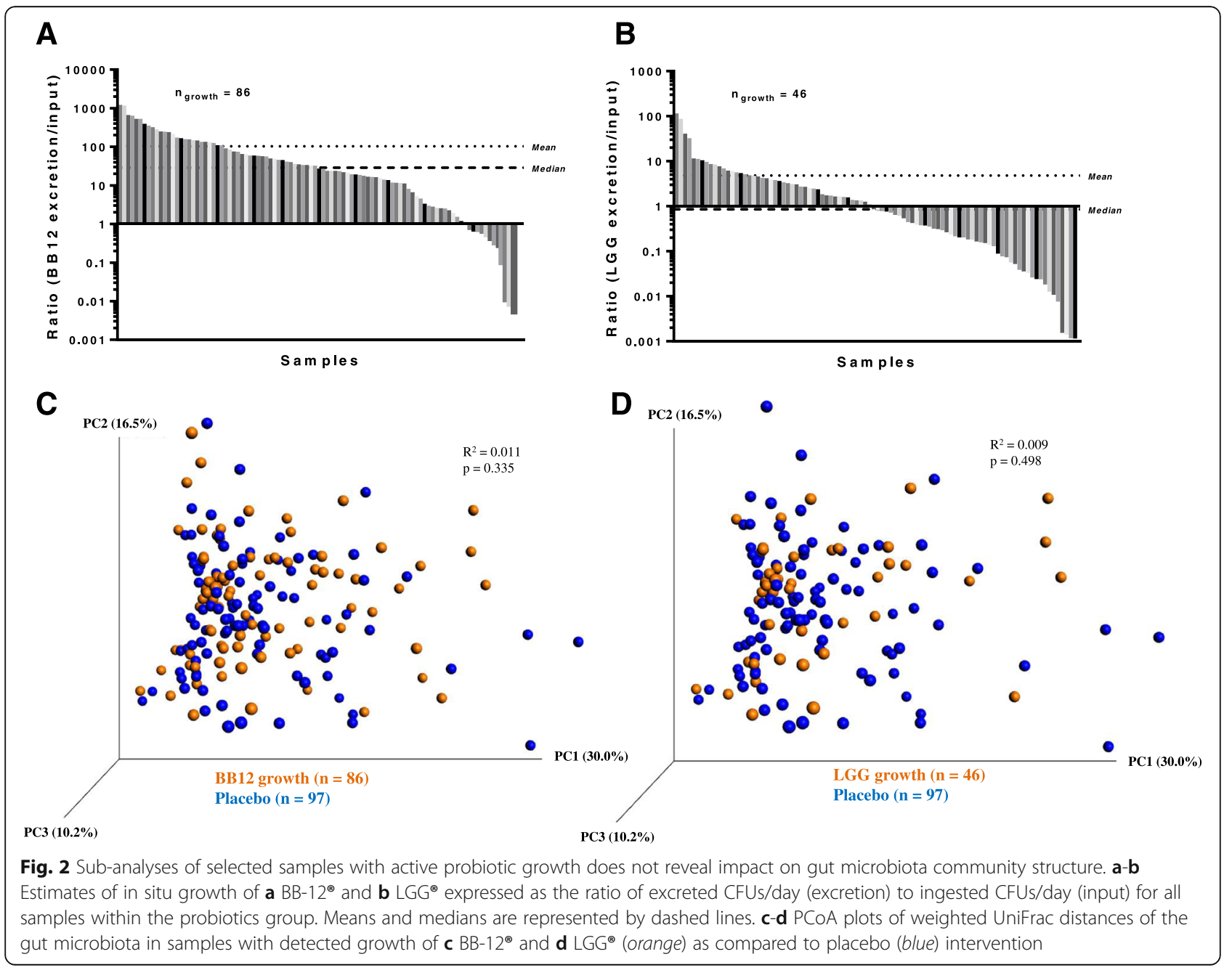


addressed gut microbiota development in the offspring at ages $10 \mathrm{~d}, 3 \mathrm{~m}, 1 \mathrm{y}$ and $2 \mathrm{y}[21,26]$. While significant mother-to-infant transmission of $\mathrm{LGG}^{\circ}$ (but not of BB$12^{\circ}$ or $\mathrm{LA}-5^{\circ}$ ) is reported, no effects on infant gut microbiota composition or diversity were found [21, 26], which is in line with our findings. Similarly, oral administration of B. bifidum W23, B. lactis W52 and Lactococcus lactis W58 during the last 6 weeks of pregnancy and subsequently to offspring at high risk of atopic disease during the first year of life is reported to have no effect on the endogenous infant gut microbiota composition and diversity, neither during the intervention nor at a later follow-up [27]. Also, 8 weeks daily administration of either $L$. acidophilus NCFM or B. lactis Bi-07 to infants aged 7-24 months and diagnosed with atopic dermatitis is reported not to change the composition and diversity of the main fecal bacterial populations as compared to a placebo group [28]. While these studies together strongly suggest that exposure to commonly applied (non-endogenous) probiotics in early life does not interfere with the natural succession and development of the infant gut microbiota, they do not consider the fact that probiotics may not colonize or grow efficiently in all individuals. In the present study, we thus further conducted sub-analyses stratifying individuals based on estimates of probiotic proliferation of the given strain after ingestion, but still found no support of the hypothesis that ingestion of the given probiotics alter the endogenous gut microbiota. Although our assessment may have underestimated the number of samples characterized by proliferation of the ingested probiotics, the selected samples with excretion/intake ratios above 1 represent the most extreme examples and would be expected to have shown an impact on gut microbiota composition if this had been affected.

\section{Conclusion}

We show that alteration of the early life gut microbial community was not achieved by oral administration of $\mathrm{LGG}^{\circ}$ and $\mathrm{BB}-12^{\circ}$ despite proliferation of the ingested probiotics in the infant intestine. We thus conclude that modulation of the endogenous microbiota is unlikely to be the causal mechanism behind putative effects of these specific probiotic strains on infant health. However, this does not exclude a direct impact of the strains on the host intestinal barrier function, gut associated immune responses and/or systemic metabolic effects, which may or may not depend on probiotic proliferation.

\section{Additional file}

Additional file 1: Table S1. Primers used to detect and quantify BB-12 ${ }^{\oplus}$ and $\mathrm{LGG}^{\oplus}$. Table S2. Strains used for evaluation of primer specificity. Table S3. OTUs with differential abundance comparing baseline samples. Table S4. OTUs with differential abundance comparing samples with BB- $12^{\circledast}$ growth to placebo. Table S5. OTUs with differential abundance comparing samples with $\mathrm{LGG}^{\oplus}$ growth to placebo. Figure S1. Gut microbiota changes over the course of the 6 months intervention. Figure S2. Gut microbiota at baseline does not differ between probiotics and placebo groups. Figure S3. Evaluation of primer specificity for $\mathrm{LGG}^{\circledR}$ and $\mathrm{BB}-12^{\circledR}$ primers. Figure S4. Gut microbial alpha diversity measures are not affected by active growth of probiotics. (PDF $532 \mathrm{~kb}$ )

\section{Abbreviations}

CFU: Colony forming units; FDR: False Discovery Rate; OTU: Operational Taxonomic Unit; PCoA: Principal coordinate analysis; qPCR: Quantitative polymerase chain reaction

\section{Acknowledgements}

We thank the participating infants and their families for their contribution to the study.

\section{Availability of data and materials}

Sequencing data are available at NCBI Sequence Read Archive with the Accession Number SRP100762 under BioProject PRJNA360073.

\section{Funding}

The study was funded by Innovation Fund Denmark, grant number 0603-00579B (ProbiComp) given to KFM and 0603-00487B (Center for Gut, Grain and Greens) given to TRL. Additionally, funding was provided by University of Copenhagen and Chr. Hansen AVS.

\section{Authors' contributions}

KFM and AL designed the intervention study. Intervention trial, data and fecal collection from participants and data analyses on study participant characteristics were obtained by RPL. MFR performed 16S rRNA amplicon library preparation and specific GPCR. MFR, MIB \& TRL analyzed and interpreted the data. MFR wrote the paper. All authors read and approved the final manuscript.

\section{Ethics approval and consent to participate}

The study protocol was approved by the Committees on Biomedical Research Ethics for the Capital Region of Denmark (H-4-2014-032), and the study was registered at clinicaltrials.org (NCT02180581) at June 30, 2014. The first participant was enrolled August 22, 2014. All parents signed a consent form.

\section{Consent for publication}

Not applicable.

\section{Competing interests}

Chr. Hansen A/S provided additional funding for the project, but had no influence on the interpretation of the data.

\section{Publisher's Note}

Springer Nature remains neutral with regard to jurisdictional claims in published maps and institutional affiliations.

\section{Author details}

${ }^{1}$ National Food Institute, Technical University of Denmark, Kemitorvet, DK-2800 Lyngby, Denmark. Department of Nutrition, Exercise and Sports, University of Copenhagen, Rolighedsvej 26, DK-1958 Frederiksberg C, Denmark.

Received: 14 June 2017 Accepted: 11 August 2017

Published online: 17 August 2017

\section{References}

1. WHO. Health and Nutritional Properties of Probiotics in Food including Powder Milk with Live Lactic Acid Bacteria. 2001; October:1-34.

2. Collins MD, Gibson GR. Priobiotics, prebiotics, and symbiotics: approaches for modulating the microbial ecology of the gut. Am J Clin Nut. 1999; 69(suppl):1052S-7S.

3. Hemarajata P, Versalovic J. Effects of probiotics on gut microbiota: mechanisms of intestinal immunomodulation and neuromodulation. Therap Adv Gastroenterol. 2013;6:39-51. 
4. Putignani L, Del Chierico F, Petrucca A, Vernocchi P, Dallapiccola B. The human gut microbiota: a dynamic interplay with the host from birth to senescence settled during childhood. Pediatr Res. 2014;76:2-10.

5. Kristensen NB, Bryrup T, Allin KH, Nielsen T, Hansen TH, Pedersen O. Alterations in fecal microbiota composition by probiotic supplementation in healthy adults: a systematic review of randomized controlled trials. Genome Med. 2016;8:52.

6. Brüssow H. How stable is the human gut microbiota? And why this question matters. Environ Microbiol. 2016;18:2779-83.

7. Rodríguez JM, Murphy K, Stanton C, Ross RP, Kober Ol, Juge N, et al. The composition of the gut microbiota throughout life, with an emphasis on early life. Microb Ecol Health Dis. 2015;26:26050.

8. Laursen RP, Larnkjær A, Ritz C, Hauger H, Michaelsen KF, Mølgaard C. Probiotics and child care absence due to infections: a randomized controlled trial. Pediatrics. 2017;140:e20170735.

9. Edgar RC. UPARSE: highly accurate OTU sequences from microbial amplicon reads. Nat Methods. 2013;10:996-8.

10. Caporaso JG, Kuczynski J, Stombaugh J, Bittinger K, Bushman FD, Costello EK, et al. QIIME allows analysis of high-throughput community sequencing data. Nat Methods. 2010;7:335-6.

11. Wang Q, Garrity GM, Tiedje JM, Cole JR. Naive Bayesian classifier for rapid assignment of rRNA sequences into the new bacterial taxonomy. Appl Environ Microbiol 2007;73:5261-5267. doi:10.1128/AEM.00062-07.

12. DeSantis TZ, Hugenholtz P, Larsen N, Rojas M, Brodie EL, Keller K, et al. Greengenes, a chimera-checked 165 rRNA gene database and workbench compatible with ARB. Appl Environ Microbiol. 2006;72:5069-72.

13. Altschul SF, Gish W, Miller W, Myers EW, Lipman DJ. Basic local alignment search tool. J Mol Biol. 1990;215:403-10.

14. Huson DH, Scornavacca C. Dendroscope 3: an interactive tool for rooted phylogenetic trees and networks. Syst Biol. 2012;61:1061-7.

15. Brandt $K$, Alatossava T. Specific identification of certain probiotic Lactobacillus rhamnosus strains with PCR primers based on phage-related sequences. Int J Food Microbiol. 2003;84:189-96.

16. Endo A, Aakko J, Salminen S. Evaluation of strain-specific primers for identification of Lactobacillus rhamnosus GG. FEMS Microbiol Lett. 2012;337:120-5.

17. Briner AE, Lugli GA, Milani C, Duranti S, Turroni F, Gueimonde M, et al. Occurrence and diversity of CRISPR-Cas systems in the genus bifidobacterium. PLoS One. 2015;10:e0133661.

18. De Man JC, Rogosa M, Sharpe ME. A medium for the cultivation of lactobacilli. J Appl Bacteriol. 1960;23:130-5.

19. Benjamini Y, Hochberg Y. Controlling the false discovery rate - a practical and powerful approach to multiple testing. J R Stat Soc Ser B-Methodological. 1995;57:289-300

20. Laursen MF, Andersen LBB, Michaelsen KF, Mølgaard C, Trolle E, Bahl Ml, et al. Infant gut microbiota development is driven by transition to family foods independent of maternal obesity. mSphere. 2016;1:e00069-e00015. doi:10.1128/mSphere.00069-15.

21. Avershina E, Lundgård K, Sekelja M, Dotterud C, Storrø O, Øien T, et al. Transition from infant- to adult-like gut microbiota. Environ Microbiol. 2016;18:2226-36.

22. Martin R, Makino H, Cetinyurek Yavuz A, Ben-Amor K, Roelofs M, Ishikawa E, et al. Early-life events, including mode of delivery and type of feeding, siblings and gender, shape the developing gut microbiota. PLoS One. 2016;11:e0158498.

23. Burkitt D, Morley D, Walker A. Dietary fibre in under- and overnutrition in childhood. Arch Dis Child. 1980;55:803-7.

24. Myo-Khin, Thein-Win-Nyunt, Kyaw-Hla S, Thein-Thein-Myint, Bolin TD. A prospective study on defecation frequency, stool weight, and consistency. Arch Dis Child. 1994;71:311-3-4.

25. Lozupone CA, Stombaugh JI, Gordon JI, Jansson JK, Knight R. Diversity, stability and resilience of the human gut microbiota. Nature. 2012;489:220-30.

26. Dotterud CK, Avershina E, Sekelja M, Simpson MR, Rudi K, Storrø O, et al. Does maternal perinatal probiotic supplementation alter the intestinal microbiota of mother and child? A randomized controlled trial. J Pediatr Gastroenterol Nutr. 2015;61:200-7.

27. Rutten NBMM, Gorissen DMW, Eck A, Niers LEM, Vlieger AM, der Vaart I, et al. Long term development of gut microbiota composition in atopic children: impact of probiotics. PLoS One. 2015;10:1-17.

28. Larsen N, Vogensen FK, Gøbe R, Michaelsen KF, Abu Al-Soud W, Sørensen $\mathrm{SJ}$, et al. Predominant genera of fecal microbiota in children with atopic dermatitis are not altered by intake of probiotic bacteria Lactobacillus acidophilus NCFM and Bifidobacterium animalis subsp.lactis Bi-07. FEMS Microbiol Ecol. 2011;75:482-96.

\section{Submit your next manuscript to BioMed Central and we will help you at every step:}

- We accept pre-submission inquiries

- Our selector tool helps you to find the most relevant journal

- We provide round the clock customer support

- Convenient online submission

- Thorough peer review

- Inclusion in PubMed and all major indexing services

- Maximum visibility for your research

Submit your manuscript at www.biomedcentral.com/submit 